Service social

\title{
Signification et conséquences sociales des changements technologiques
}

\section{Réjean Mathieu}

Volume 36, numéro 1, 1987

L’informatique dans les services sociaux

URI : https://id.erudit.org/iderudit/706344ar

DOI : https://doi.org/10.7202/706344ar

Aller au sommaire du numéro

\section{Éditeur(s)}

École de service social de l'Université Laval

ISSN

1708-1734 (numérique)

Découvrir la revue

Citer cet article

Mathieu, R. (1987). Signification et conséquences sociales des changements technologiques. Service social, 36(1), 119-128. https://doi.org/10.7202/706344ar
Résumé de l'article

L'auteur pose le problème général de l'émergence et du développement des nouvelles technologies : malgré le progrès indéniable qu'elles amènent, certains effets négatifs doivent aussi être pris en considération si on veut en situer les possibilités et les limites.

Il remet en question la conception prédominante de l'efficacité, d'où il découle des catégories de gens qui réussissent alors que d'autres sont rejetées par le système parce qu'elles ne répondent pas aux nouveaux critères d'efficacité. Bref, l'émergence des nouvelles technologies demande un véritable débat quant à leurs impacts sociaux. On peut ici penser à des dimensions comme la confidentialité, le contrôle possible par l'accès aux informations, ou les conséquences sur le monde du travail. Il faut aussi prévoir que ces changements auront une influence plus marquante sur les populations que desservent les travailleurs sociaux. 


\section{Signification et conséquences sociales des changements technologiques}

\section{Réjean Mathieu}

De l'informatique à l'intelligence artificielle, en passant par la robotique, la bureautique et les autres applications des nouvelles technologies caractéristiques de la période actuelle, tous ces développements scientifiques fascinent par les perspectives qu'ils ouvrent; ceci est plus évident dans des domaines comme les tâches secrétariales, la recherche, la rédaction de textes, les programmes informatiques en général, et enfin, les systèmes «à base de connaissances " qui permettent les systèmes experts qu'utilisent les chercheurs dans le domaine de l'intelligence artificielle. Que ce soit pour accélérer grandement la réalisation de certaines tâches ou pour tenter de faciliter la solution de problèmes de divers ordres (prise de décision, traduction, etc.), il est indéniable qu'il faut développer l'utilisation de ces technologies de pointe aussi bien en travail social qu'ailleurs. Car elles peuvent être utiles, non seulement à nous comme travailleurs professionnels, mais aussi aux usagers et usagères de nos "services".

Ceci suppose cependant que l'utilisation que nous en ferons soit conséquente à nos objectifs et respectueuse des caractéristiques des personnes qui reçoivent ces services. En effet, si nous, en travail social, ne sommes pas conscients-tes des possibles effets négatifs de ces mêmes technologies, qui donc le sera? Si nous ne portons pas une attention particulière à la nécessité de privilégier d'abord l'humain et ses rapports et de développer un apport technologique qui soit subordonné à cette primauté, qui donc le fera?

C'est en ce sens, et non pour empêcher le progrès indéniable que peuvent constituer ces technologies nouvelles, qu'il est bon d'aborder la réflexion proposée ici : dégager divers effets douteux ou même négatifs que certaines conceptions du " virage technologique " peuvent créer afin d'en mieux situer, à la fois les possibilités et les limites. 
Peut-être pourrons-nous également, dans une approche lucide et critique, trouver le moyen d'utiliser ces technologies au maximum, dans des visées et des perspectives les plus favorables possible aux intérêts des usagers des services sociaux. On peut affirmer qu'il s'agit d'une approche "moralisatrice"; sans jouer sur les mots, ne pourraiton pas dire plutôt qu'elle est stratégique?

\section{De l'engouement à l'utilisation réfléchie}

Quand le discours social dominant parle de "virage technologique nécessaire ", de "nouvelles technologies à intégrer » ou utilise d'autres slogans similaires, il reflète à la fois le besoin de restructuration du capital au sortir d'une crise et une foi quasi magique et à courte vue dans la science.

En effet, ce discours n'est pas nouveau. II refait surface après chaque crise économique importante puisqu'il manifeste la recherche d'une solution évolutive au marasme industriel et au retard des structures économico-politiques. On tentera donc de faire des nouvelles technologies, quelles qu'elles soient, le fer de lance de la relance économique.

Dans la même foulée, sans trop s'interroger sur les effets sociaux qu'elle entraînera, on fait un acte de foi dans cette nouvelle technologie, sur la base d'une conception traditionnelle de la science, conception qui la voit comme un apport «neutre, bienveillant et sûr » à la vie sociale. En effet, les méthodes préconisées par la science, le ton rigoureux et systématique qu'elle adopte, le discours semé de concepts rassurants qu'elle tient, comme l'objectivité, le savoir, la connaissance, la vérité, tout cela tend à la situer au-delà des influences économiques et politiques, au-delà de la sphère du culturel même...

Mais cette idéologie scientifique, sans lui opposer un retour aux magiciens et à la religiosité, tend à disparaître de plus en plus, même en milieu scientifique. Si une grande rigueur épistémologique demeure comme objectif à atteindre, on est de plus en plus conscient du fait que la science ne se situe pas dans un " au-delà " quasi divin, qu'elle est ellemême déterminée dans ses priorités, dans sa démarche et même dans ses résultats par un "bloc » économico-politique particulier. Les besoins industriels, militaires et économiques en général l'influencent profondément. D'autant plus que la recherche subventionnée est elle-même pensée pour répondre à des fins militaires (plus de la moitié) ou à des exigences de développement industriel.

En ce sens, on est loin du cybernéticien d'Aurel David qui " reste enfermé dans la technique ». ${ }^{1} \mathrm{Si}$ (en apparence) il ne touche pas à la morale, si seuls "les moyens sont de sa compétence», il n'est souvent 
qu'un instrument sans liberté profonde au service d'impératifs militaires ou économiques. Même les débuts de la cybernétique, au Massachusett's Institute of Technology avant la deuxième guerre mondiale, ont puisé leurs objectifs dans la recherche d'armes automatiques "plus efficaces que les humains". On voit ainsi que, si le discours technologique glorifie l'avènement d'une ère nouvelle où le "travail machinal " sera confié aux machines libérant ainsi l'homme pour créer, la réalité n'est pas toujours aussi simple. Si la machine peut effectivement libérer I'homme de certaines tâches, elle intervient souvent dans d'autres desseins. Et parmi ceux-ci, s'il y en a qui ont un caractère industriel et militaire, il en est un plus fondamental encore qui les sous-tend: l'efficacité! Ou plutôt, une certaine conception de l'efficacité.

Or, précisément, cette conception correspond à une des caractéristiques fondamentales de la société contemporaine : la technocratie, en particulier comme idéologie. Cette idéologie, que d'aucuns qualifient de "cybernétiste", tend à considérer la société comme une machine qu'il s'agit de rendre plus efficace en utilisant les techniques appropriées pour résoudre les problèmes. Ainsi, l'efficacité, en particulier celle de la production, prime sur toute considération morale ou sociale qu'on laissera, comme n'étant pas de sa compétence, aux religions ou aux "spécialistes " du travail social ou psychologique. Le concept clé devient la planification, laquelle, dans la mesure où elle n'est pas autogérée, devient une sorte de rouleau compresseur conçu pour assurer la plus grande efficacité possible et pour délester le monde de la production de ses "poids morts" avec le moins de répercussions sociales possible.

On se retrouve ainsi dans la situation où l'instrument de l'efficacité tend à devenir l'objectif lui-même, l'ensemble des forces sociales actives et des intérêts individuels convergeant vers la réalisation maximale du " plan », et vers la reproduction des cadres et rapport sociaux existants. En conséquence, des technologies nouvelles, qui pourraient servir des perspectives d'avenir réellement progressistes deviennent des instruments au service "d'orientations préalables de valeurs", ${ }^{2}$ d'intérêts et de cultures de classes ou de couches spécifiques, puisque ce sont ces valeurs qui déterminent, au bout du compte, la sélection même des conditions techniques et leur utilisation. Le système en place a subi une chirurgie esthétique et en ressort plus fort, au moins jusqu'à sa prochaine crise structurelle.

Il y a une nuance de taille cependant : ce changement de visage a comme effet de transformer les gens eux-mêmes dans la mesure où certaines règles du jeu sont changées et où, pour se "réaliser ", il faut maintenant s'inscrire dans les nouvelles structures mentales exigées par les nouvelles technologies. D'où un nouveau type de gens qui ont 
"réussi », comme aussi de nouvelles catégories rejetées par le système parce que ne répondant pas aux nouveaux critères d'efficacité. De nouvelles normes sociales apparaissent, souvent plus fortes encore que les normes antérieures, et tendent à accroître le nombre et la diversité des " marginalités ». En même temps que se développera la "classe " des technocrates, et que même la bourgeoisie aura à se "technocratiser" pour devenir véritablement contemporaine, de nouvelles catégories de gens seront "déclassées " ne jouissant ni de la propriété juridique des moyens de production, ni de leur "possession de fait " (contrôle technocratique), et n'étant pas en mesure de s'inscrire de façon satisfaisante dans ces nouveaux réseaux ou de survivre à la concurrence et à la monopolisation qui continueront de caractériser le système.

On en vient donc à affirmer à la fois que la science et la technique agissent aujourd'hui comme idéologie ${ }^{3}$ et qu'elles ne sont, en ellesmêmes, ni bonnes ni mauvaises, mais "en tension vers la vérité "... ${ }^{4}$

\section{Concrètement, des constantes}

Même si elles sont étroitement imbriquées les unes dans les autres, on peut quand même distinguer un certain nombre de constantes du point de vue technique d'une part, et d'un projet de société à construire d'autre part. En ce qui a trait à la technique, il suffit de se rappeler les discours de relance de nos divers paliers gouvernementaux pour reconnaître le lien étroit entre les nouvelles technologies et l'ensemble des stratégies économiques et industrielles, en particulier des projets de relance. Dans le cas du Québec, il apparaît qu'un accent important est mis sur la biotechnologie et l'informatique. Pour en assurer le développement, on tentera de former de nouveaux chercheurs dans ces domaines et de développer une recherche liée à la production industrielle dans ces secteurs.

En ce qui a trait au projet collectif d'une nouvelle société à faire naître, on prévoit l'apprentissage des nouvelles technologies dans les écoles, le développement de la bureautique dans les milieux de travail, etc.

Par contre, on est généralement peu attentif aux effets sociaux prévisibles de ce virage, tant au niveau des libertés individuelles qu'aux plans culturel et social. Ce hiatus provient, encore une fois, de la croyance spontanée dans les "vertus » de la science et de la technologie : c'est le progrès !

Bref, à ce projet de société à construire en intégrant au maximum les nouvelles technologies, sans véritable approche critique, on se doit d'opposer un autre projet : la "négociation " du virage technologique. 
Cela suppose cependant le développement d'un véritable débat démocratique sur les technologies nouvelles et leurs effets, comme aussi une attention constante car elles ne sont pas qu'un "danger pour la société ", et il est essentiel de savoir les intégrer dans la mesure où elles serviront à l'amélioration de la qualité des services, à une meilleure communication, à une information plus rapide et plus adéquate, etc. Ainsi, il faudrait encourager la mise sur pied ou le développement d'organisations regroupant les usagers ayant des intérêts communs ou mandatées pour défendre ces intérêts.

\section{Des effets majeurs sur toute la société}

Si la science et la technologie sont des outils potentiellement utiles pour comprendre la réalité, elles sont également encadrées dans des "blocs historiques", culturels et institutionnels : elles ne sont donc pas neutres et si elles ont une réelle efficacité, il faut se questionner sur la nature de cette efficacité et se demander s'il y a vraiment progrès.

Face aux effets négatifs possib́les, il faudra en particulier développer une grande vigilance autour des libertés fondamentales. Par exemple, les nouvelles technologies rendent possibles de nombreuses atteintes aux droits individuels et nous devrons prendre les mesures nécessaires pour que la "personne privée " puisse contrôler les informations personnelles que réseaux institutionnels et entreprises pourraient avoir à son sujet. Les mémoires fabuleuses des ordinateurs, l'accès à ces mémoires rendu possible à distance par la télématique, leur utilisation à l'école, à l'usine, dans les loisirs, à la maison, mais aussi à la guerre, en médecine et dans toutes les officines de l'État, ne peuvent que rendre très difficile le contrôle sur les informations privées que ce système aux ramifications multiples pourrait emmagasiner et transmettre. Ce système touche à tout, est partout et, par lui, l'État, par exemple, pourrait recueillir une infinité d'informations sur le plus grand nombre d'invididus, y faire des recoupements quasi illimités, etc. Qu'on pense aux centaines de milliers de dossiers de la Gendarmerie royale du Canada, aux dossiers des banques, aux "listes noires" des associations de propriétaires; qui utilisera ces données? Plus encore, quelle est la nature de ces données? Il est tout à fait possible qu'elles aient un caractère subjectif, raciste, sexiste, etc. ; mais une fois dans la machine, elles acquièrent une sorte de "statut" objectif fort dangereux! De nombreux droits risquent d'être atteints, dont celui à la vie privée n'est pas le moindre... Mais il y a aussi des risques pour le droit à la liberté de conscience, d'expression, d'association, en raison des utilisations possibles des données et des recoupements entre, par exemple, le Ministère des affaires sociales, le 
Ministère du revenu, le Ministère de la justice et la Gendarmerie royale du Canada.

David H. Flaherty, professeur à l'Université Western Ontario et expert mondialement reconnu en matière de protection des renseignements personnels, déclarait récemment que les démunis sont les plus vulnérables aux intrusions dans leur vie privée par des organisations sociales se voulant de plus en plus efficaces; à son avis, à l'approche de l'an 2000, la protection des données est aussi importante que la question nucléaire ou la sauvegarde de l'environnement. II s'inquiétait de cette " réduction de l'espace privé ", déplorait le peu d'agressivité des agences de protection des données, allant même jusqu'à dire qu'elles "berçent l'opinion d'un sentiment de sécurité illusoire». Or, selon lui, des administrations particulièrement menaçantes à ce niveau, comme l'impôt, le bien-être social et la police, " développent des stratégies de résistance " face aux agences de protection des données. On en est au point de ne plus se préoccuper du droit à la vie privée dès lors qu'on peut se cacher sous des "priorités" comme la sécurité nationale, l'expansion des toxicomanies, les évasions fiscales, etc. Encore une fois, dans ce contexte, David H. Flaherty ajoute : "Ce sont toujours les mêmes qui trinquent : les prisonniers, les bénéficiaires d'aide sociale, les marginaux ; l'élite est plus ou moins épargnée ${ }^{5}{ }^{5} \mathrm{Or}$, ces plus vulnérables sont les populations que nous sommes censés aider à améliorer leurs conditions de vie et "l'élite épargnée", nous en faisons partie. D'où l'importance encore plus grande d'une approche particulièrement lucide et critique.

Mais, à un autre niveau, on risque plus encore : la rigidité relative du système binaire à la base de l'informatique et sa difficulté à nuancer ouvrent la porte à une normalisation encore plus importante dans notre société : ainsi, pour programmer les ordinateurs, il faudra déjà fixer des normes, lesquelles deviendront par la suite la "normale ", en dehors de laquelle on sera un "déviant", un "anormal "... Les marginaux ne risquent-ils pas de devenir plus nombreux? Mais aussi plus réprimés? Ne peut-on pas craindre un retour à une société rigide, plus normative, plus répressive, sous l'égide d'une nouvelle classe de "définisseurs de normalité " ? On me signalera qu'il est possible de pallier aux dangers de ce "système binaire»... C'est en partie vrai seulement. On n'a qu'à penser aux difficultés rencontrées par les promoteurs de l'intelligence artificielle qui, pour établir un programme pour un jeu complexe comme les échecs, par exemple, doivent reculer devant l'approche systématique qui consisterait à énumérer tous les coups possibles. Pourquoi? Parce que "le nombre de possibilités croît de manière exponentielle: c'est ce que l'on appelle l'explosion combinatoire $\#{ }^{6}$ C'est pourquoi ils chercheront plutôt à développer des "systèmes à 
base de connaissances", conduisant aux "systèmes experts", plus "nuancés", mais également limités à "des domaines d'expertise très pointus ". ${ }^{7}$ Imaginez maintenant ce que pourrait représenter, comme "explosion combinatoire", l'approche systématique qui énumérerait toutes les possibilités dans un domaine aussi complexe que le travail social ; tout en développant des systèmes experts qui pourraient être utiles dans des sphères très précises de notre travail, il faudra éviter les approches trop «classificatoires ». Or, ces approches, les services administratifs ne les adoptent-ils pas spontanément?

Au niveau des conditions de travail, les effets sont également énormes, en particulier si on se situe dans le cadre des compressions budgétaires. On peut déjà constater un certain abandon des programmes et services de prévention dans le réseau public, le transfert vers le secteur privé de certains services jugés "non urgents", etc. Bien sûr, tout cela ne relève pas seulement des nouvelles technologies, mais celles-ci n'y sont pas étrangères non plus par leur tendance à développer encore plus les normes. On peut également appréhender une certaine "taylorisation " ${ }^{8}$ du travail social, par exemple : la chaîne de montage appliquée à l'humain. À chaque étape, le temps de travail serait alors calculé selon des normes précises, comme déjà dans les hôpitaux on "suggère fortement» de ne pas prendre plus de tant de minutes pour laver un patient.

Enfin, c'est toute la société qui sera marquée par une nouvelle façon de penser, d'apprendre, de conceptualiser peut-être; et si ce n'est pas nécessairement négatif, on doit au moins tenter de prévoir les effets de cette mutation, faire des choix!

\section{Des effets inégaux}

Autant les uns, pour des intérêts et des "cultures" de classe, de couche ou de sexe, s'impliqueront dans les nouvelles technologies et les utiliseront, autant les autres, dans la même mesure et pour des raisons contraires liées aux mêmes facteurs, auront peu de propension à s'intégrer dans le réseau technocratique.

En d'autres mots, nous assistons déjà, conséquemment à une restructuration du capital, à une nouvelle division du travail. De nouvelles " compétences » apparaissent, résultats de nouveaux types d'apprentissage. Il s'agit en fait d'une nouvelle forme de contrôle qui s'exerce et se réalise " dans les chaînes opératoires d'un appareil ». ${ }^{9}$ Ainsi, on assistera peu à peu à une intégration graduelle par des couches, classes ou sexes, de l'idéologie technocratique, intégration ou " intériorisation " nécessaire, comme nous le disions plus haut, pour se "réaliser», tandis que d'autres 
ne le pourront pas et que toutes ces catégories plus ou moins nouvelles seront dominées, contrôlées par la technocratie et ses bailleurs de fonds.

Mais comment s'opérera cette division nouvelle du travail? En particulier dans une conjoncture économique où même une société aussi riche que les États-Unis appréhende, non seulement une récession, mais même une "dépression " semblable à celle de $1929^{10}$ alors que $1 \%$ des familles possédaient $36.3 \%$ de la richesse nationale (34.3\% en 1983), par opposition à $20.8 \%$ en 1949 , en période de développement économique... ? Cette nouvelle division du travail ne pourra s'opérer qu'à partir des forces socialement actives, de la même manière qu'au moment de la Révolution tranquille, c'est-à-dire par l'action conjuguée de l'État, des corporations professionnelles, de l'université et de l'école en général, ainsi que par les nouvelles structures technocratiques et les perspectives d'emploi. C'est précisément là qu'une unité de formation universitaire en travail social doit intervenir puisque, de par notre fonction même, nous pouvons être un point de rencontre des classes, couches et sexes qui auront tendance à intérioriser l'idéologie technocratique d'une part (par notre présence en milieux institutionnels, académiques et pratiques) et de ceux qui, d'autre part, lui opposeront un refus et en seront les victimes (par notre relation aux "clientèles » et notre présence dans les réseaux alternatifs).

Pour y parvenir, il nous faudra évidemment avoir une très bonne compréhension de la réalité socio-économique et des caractéristiques culturelles de ces "clientèles et des milieux alternatifs", d'autant plus que c'est précisément l'ensemble de ces caractéristiques qui les amèneront à une attitude de refus face à l'idéologie technocratique. En ce sens, c'est même cette compréhension et cette connaissance qui peuvent nous permettre d'identifier déjà, jusqu'à un certain point, la composition sociale des catégories qui ne seront pas "libérées" par les nouvelles technologies mais qui, au contraire, risquent d'en subir la domination.

En effet, l'accès à ces nouvelles technologies est et sera plus facile pour les classes dominantes et les pays les plus développés. Leur contrôle et leur développement se retrouvent une fois de plus entre les mains de quelques puissantes multinationales et de quelques pays plus aptes à s'investir dans la restructuration du capital qu'elles impliquent. Conséquemment, les pays en voie de développement et les classes défavorisées resteront aux prises avec des problèmes élémentaires comme l'analphabétisme, la malnutrition, etc. À l'intérieur même des pays développés, il est prévisible que le fossé s'élargisse entre deux catégories d'emplois : les emplois spécialisés et ceux qui ne le seront pas. Les conditions de vie et de travail des non-spécialistes risquent 
même de se détériorer, d'autant plus que le chômage ne semble guère en voie de se résorber.

La bureautique et l'automatisation en milieu de travail créeront du chômage ainsi que d'énormes problèmes de recyclage pour les gens qui ne seront pas mis à pied. Même s'il y avait création de nouveaux emplois pendant un certain temps, il faut prévoir, qu'à moyen et à long terme, on devra faire face à un chômage accru.

Les femmes en particulier subiront vraisemblablement les contrecoups de ce "virage ", compte tenu de leur importance numérique dans les emplois subalternes de bureau et dans l'ensemble des services publics. Ce sera encore plus sérieux, possiblement, quant aux femmes qui resteront à la maison, puisque leur vision du monde et leur culture auront de plus en plus tendance à se situer aux antipodes des nouvelles technologies : plus que jamais reléguées à la "vie privée", elles y seront isolées et dominées à la fois, et auront donc plus de difficultés à s'intégrer dans un monde technocratique, en particulier si elles tentent de revenir sur le marché du travail. Ajoutons que déjà, près de $80 \%$ de la "clientèle " des services sociaux est composée de femmes.

Enfin, plus les nouvelles technologies se développent, plus on peut constater que le monde devient un "grand village" traversé par une multitude d'informations mais, en même temps, ce phénomène tend à isoler les gens les uns des autres, à les influencer graduellement et à les contrôler chez eux, dans leur maison ou appartement.

Tout cela nous amène à constater une fois de plus l'urgence de " négocier » le virage technologique au lieu de le prendre sans discernement au nom du progrès. Par exemple, les conventions collectives devront de plus en plus tenir compte du recyclage, élaborer des clauses permettant de conserver un certain contrôle sur les conditions de travail, favoriser l'information et les débats sur l'impact des nouvelles technologies, revendiquer que le personnel soit informé à l'avance de tout changement technologique important, obtenir la réduction du temps de travail sans baisse de salaire, ainsi que le recyclage des employés de certains services et le maintien d'un plancher minimum d'emplois, etc. Enfin, les loisirs ou « temps libres " devront être repensés de façon créatrice, dans l'éventualité où ils occuperaient plus de place dans nos vies. ${ }^{11}$

De façon plus générale, il est clair qu'il faudra développer beaucoup de recherches sur ces différents aspects du virage technologique, créer de nouveaux lieux de débat et de pression en ce qui concerne le respect des droits fondamentaux de la personne et des collectivités, et enfin, repenser la formation universitaire afin qu'elle tienne compte de ce virage et de ses effets. 


\section{Notes}

1 David, Aurel, La cybernétique et l'humain, Paris, Gallimard, 1965, p. 15. ("Idées".)

2 SIMARD, Jean-Jacques, Québec \& Frères, inc. : la cybernétisation du pouvoir au Québec, Association Canadienne des sociologues et anthropologues de langue française, Colloque 1979, Montréal, Albert Saint-Martin, 1980, p. 362.

3 Habermas, Jürgen, La technique et la science comme idéologie, Paris, Gallimard, 1973.

4 CARON, Joseph, Communication à la table-ronde Virage technologique et/ou travail social, Semaine des sciences, Université du Québec à Montréal, 7 novembre 1983.

5 Allocution de David $\mathrm{H}$. Flaherty à la Conférence internationale des commissaires à la protection de l'information, tenue à Québec, telle que rapportée par Carole Beaulieu, Le Devoir, mardi le 22 septembre 1987, p. 3.

6 Dufay, Bruno, L'intelligence artificielle, Monaco/Paris, Éditions du Rocher/ Jean-Paul Bertrand éditeur, 1987, p. 12. ("Sciences et Découvertes »))

7 Ibid.

8 Tendance à développer un processus d'organisation du travail axé sur des techniques plus " efficaces» et plus rentables, telles que le chronométrage, la transmission par courroie, etc. Ainsi nommée d'après l'économiste américain Frédérick W. Taylor (1856-1915).

9 SimARD, Jean-Jacques, op. cit., p. 363.

10 Voir le dossier: "How ripe for a crash", Time, 5 octobre 1987, pp. 40ss et le statut de "best-sellers" de plusieurs volumes portant des titres évocateurs comme The Great Depression of 1990, etc.

11 Procès-verbal de la table-ronde Virage technologique et/ou travail social, Semaine des sciences, Université du Québec à Montréal, 7 novembre 1983. 